\title{
IMPACTO AGRÍCOLA E DA PECUÁRIA NO ESTADO DO PARÁ
}

\author{
Severino Félix de Souza ${ }^{1}$ \\ Gessiane da Silva Paulino ${ }^{2}$ \\ Armando Lirio de Souza ${ }^{3}$
}

RESUMO: As políticas econômicas no geral buscam elevar o bem-estar da sociedade em questão através da distribuição e formas de fomento ao aumento de renda, por exemplo. Neste sentido, agricultura familiar é de grande importância tanto no cenário econômico como para a segurança alimentar da população, uma vez que o crescimento agrícola é capaz de constituir um importante vetor para a redução da pobreza. O objetivo deste trabalho é verificar o impacto agrícola e da pecuária na economia do estado do Pará. Para este objetivo foi usado o modelo econométrico de dados em painel. As estimações do modelo mostraram que tanto o Valor Agregado Bruto como o Produto Interno Bruto per capita apresentam impactos positivos, sendo estes maiores no Valor Agregado Bruto, ou PIB Agrícola.

Palavras-Chaves: Impacto agrícola. Pecuária. Dados em painel.

\footnotetext{
1 Graduação em Ciências Econômicas (UFCG), Mestre em Economia (UFRN) e Doutorando em Economia (UFPA). E-mail: severinofelix@gmail.com

${ }^{2}$ Graduação em Ciências Econômicas (UFPA), Mestranda em Economia (UFPA). E-mail: gessianepaulino18@gmail.com

${ }^{3}$ Doutor em Desenvolvimento Rural pelo PGDR/UFRGS. E-mail: armandolirio@gmail.com
} 


\section{INTRODUÇÃO}

As políticas econômicas, no geral, buscam elevar o bem-estar da sociedade em questão através da distribuição e formas de fomento ao aumento de renda, por exemplo. Nesse sentido, a melhoria da distribuição de renda dos indivíduos e regiões equivale a aumentar qualitativamente e distributivamente o mercado interno de bens de consumo do país (IPEA, 2014).

A agricultura familiar desempenha um papel muito importante neste cenário, assim como a pecuária, no que diz respeito à produção de alimentos a partir de suas lavouras permanentes e temporárias, e na produção de leite.

As lavouras permanentes são plantios de longa duração que não necessitam de um novo plantio após a colheita, estas são capazes de produzir por diversos períodos, diferente das lavouras temporárias que representam áreas de curta duração, menor que um ano, necessitando de novos plantios depois da colheita (FAPESPA, 2015).

Além do fator econômico, é necessário destacar também o fator alimentar e nutricional da população, uma vez que, segundo Hoffman (2014), um percentual considerável dos alimentos consumidos pela população provém da agricultura familiar e que esta tem sua importância familiar no Brasil, porém, ele ressalta que é impossível avaliar precisamente qual a parcela do que é consumido no país.

Estados como a Paraíba, por exemplo, atendem cerca de 11.300 famílias em mais de 80 municípios, na intenção de melhorar a qualidade nutricional das famílias de baixa renda através da distribuição de leite no intuito de combater a fome e à desnutrição infantil (PARAÍBA, 2017).

Em relação à cultura das lavouras permanentes do estado do Pará, segundo a FAPESPA (2015), o desempenho das culturas desse segmento é bastante diferenciado e apenas três dentre as maiores produções apresentaram variação positiva em termos de área e quantidade produzida, que são: limão, cacau e banana.

Já no que tange a cultura das lavouras temporárias, alguns produtos que tem impacto diretamente na renda do produtor familiar têm se mantido praticamente estável, como é o caso da mandioca, que nos últimos anos tem mantido sua produção estável com um pequeno crescimento de $0,4 \%$, devido principalmente ao baixo nível de 
tecnologia implantando no seu sistema de produção e redução da mão de obra familiar (FAPESPA, 2015).

Outro motivo que pode gerar diminuição do impacto econômico da agricultura dar-se pelo cenário ambiental, uma vez que a expansão da cultura da cana-de-açúcar passa por restrições devido ao Decreto Federal n 6.961 de 2009, que proíbe o plantio desta cultura nos biomas da Amazônia e Pantanal, fazendo com que esta produção concentre-se em uma pequena faixa, gerando estabilidade na área colhida. Programas institucionais do governo federal, como o Programa Nacional de Alimentação Escola (PNAE) e o Programa de Aquisição de Alimentos (PAA) surgiram como forma de buscar incrementar a economia do produtor agrícola familiar, na tentativa de diminuir o intervalo entre a produção, a comercialização e o destino final dos alimentos, como também aumentando o retorno econômico.

O PNAE tem como objetivo fazer com que os municípios absorvam pelo menos $30 \%$ da merenda escolar vinda da agricultura familiar, já o PAA, utiliza processos de comercialização que favorecem a compra direta de produtos de agricultores familiares ou de suas organizações, promovendo a agregação de valor à produção (BRASIL, 2012). Em relação à pecuária, os primeiros bovinos que chegaram ao estado do Pará, vieram do Maranhão através de vias marítimas, em Belém e Marajó, em meados de 1620 e 1680, respectivamente, espalhando-se pelo Baixo Amazonas e Nordeste paraense, é o que aponta Medeiros Neto (1970).

A pecuária do Pará era uma atividade dominantemente extensiva e de baixa produtividade, incapaz de gerar capital de maior expressão, pois os rebanhos ainda eram pequenos e tinham na pequena capacidade de pastagens e baixa disponibilidade de forragem, algumas limitações, como afirma Láu (2006). A produção leiteira é tida como atividade praticamente familiar, que desempenha papel importante com a geração de empregos e renda, no entanto, sem possuírem uma visão técnica empresarial, os produtores acabam optando por tecnologias falhas, no que se refere à alimentação, melhoramento genético e sanitário dos animais, que acaba gerando um baixo aproveitamento pelas indústrias (LÁU, 2006).

Toda essa transformação não se deu de forma tão rápida, ou seja, a partir de meados de 1960 até o final da década de 1980, houve um forte processo de 
transformação pelo qual passou a agricultura brasileira, ou seja, este setor passava a crescer a taxas expressivas e a produtividade da terra e do trabalho fazia agora parte da dinâmica do crescimento deste setor, é o que indica IPEA (2014).

Este trabalho divide-se em algumas seções, buscando demonstrar os impactos agrícolas e da pecuária na economia do estado do Pará a partir de um modelo econométrico de dados em painel. A primeira seção é uma breve introdução para contextualizar o cenário agrário do Estado do Pará. Em seguida são expostos os procedimentos metodológicos, trazendo na terceira seção os resultados e a discussão destes dados e por fim, as considerações finais.

\section{PROCEDIMENTOS METODOLÓGICOS}

A metodologia escolhida para realizar a modelagem de Dados em Painel levou em consideração o que afirma Hsiao (1986), ou seja, os modelos para dados em painel apresentam vantagens se comparados aos de corte transversal e/ou séries temporais, no que se refere ao controle da heterogeneidade.

Greene (2008) enfatiza a eficiência de uma estimação a partir dos dados em painel, apresentando vantagens como um maior número de observações, o que assegura um aumento nos graus de liberdade e redução de multicolinearidade.

No entanto, segundo Hsiao (2007), a coleta de dados em painel é muito mais dispendiosa do que a coleta de dados de séries temporais, entretanto, já existe em países desenvolvidos e em desenvolvimento, uma ampla disponibilidade destes dados.

De acordo com Duarte, Lamounier e Takamatsu (2007), o modelo geral de dados em painel é simbolizado por:

$$
y_{i t}=\beta_{0 i t}+\beta_{1 i t} x_{1 i t}+\cdots+\beta_{\text {nit }} x_{k i t}+e_{i t}
$$

Onde i representa diferentes indivíduos e t o período de tempo analisado. $\beta_{0}$ está relacionado ao intercepto e $\beta_{k}$ ao coeficiente angular correspondente a k-ésima variável explicativa do modelo.

De acordo com Bacchi e Caldarelli (2015), na metodologia avaliada, existem diversos tipos de modelos de agrupamentos de dados. São eles: 
Pooled, que é representado pela equação (1), onde se assume que os parâmetros $\beta_{0} e \beta_{1}$ são comuns para todas as unidades. $O$ método se adequa e mostra com unidades selecionadas intuitivamente, que apresentam semelhanças em suas características. O modelo pooled pode ser estimado pelos Mínimos Quadrados Ordinários (MQO), com $\operatorname{Cov} X_{i t}, u_{i t}=0$, ou seja, os erros são ruído branco e não se correlacionam com os regressores. O agrupamento de dados de séries temporais e de corte seccional tem a capacidade e a possibilidade de tratar da heterogeineidade entre as unidades, se existentes, é o que afirma Stock e Watson (2007). Nesse intuito são sugeridos dois modelos:

Efeitos fixos: este modelo controla efeitos nas variáveis omitidas, que alteram entre indivíduos e permanecem constantes ao longo do tempo. Desta forma, é possível supor que o intercepto à variável de um indíviduo para outro, porém, é constante no tempo (DUARTE, LAMOUNIER E TAKAMATSU, 2007).

Hill, Griffiths e Judge (1999) apontam as suposições do modelo como:

$$
\beta_{0 i t}=\beta_{0 i} \beta_{1 i t}=\beta_{1} \ldots \beta_{k i t} \beta_{k}
$$

Desta forma, o modelo de efeitos fixos será dado por:

$$
y_{i t}=\alpha_{i}+\beta_{1} x_{1 i t}+\cdots+\beta_{k} x_{k i t}+e_{i t}
$$

Onde, $\alpha_{i}$ detém o efeito de dimensão de países/estados/municípios, recursos naturais existentes, etc. Quando o intercepto é correlacionado com as variáveis explicativas, a modelagem de efeito fixo passa a ser a melhor alternativa para modelar os dados em painel. Outra importante suposição de acordo com Duarte, Lamounier e Takamatsu (2007), é que no modelo de efeitos fixos, o intercepto é um parâmetro fixo, desconhecido e que tem a capacidade de captar diferenças entre indivíduos que estão na amostra, portanto, a inferência realizada no modelo, é somente dos indivíduos cujos dados são dispostos.

Efeitos aleatórios: Esta modelo apresenta as mesmas suposições do modelo de efeitos fixos, ou seja, o intercepto varia de um indivíduo para o outro, mas não ao longo 
do tempo. A diferente entre os dois modelos está na forma de tratar o intercepto - no modelo fixo, o intercepto é um parâmetro fixo, no modelo aleatório, o intercepto é uma variável aleatória - (DUARTE, LAMOUNIER E TAKAMATSU, 2007).

A modelagem é representada da seguinte forma:

$$
\begin{aligned}
& y_{i t}=\left(\alpha+V_{i}\right)+\beta X_{i t}+\cdots+u_{i t} \\
& y_{i t}=\alpha+\beta X_{i t}+\cdots+\left(v_{i}+u_{i t}\right) \\
& y_{i t}=\alpha+\beta X_{i t}+\cdots+w_{i t}
\end{aligned}
$$

Onde o termo de erro pode ser decomposto em: $w_{i t}=v_{i}+u_{i t}$

Com: $v_{i} N\left(0, \sigma_{v}^{2}\right)$

$$
u_{i} N\left(0, \sigma_{u}^{2}\right)
$$

Em que os erros são homocedásticos e não autocorrelacionados. A hipótese Cov $X_{i t}, u_{i t}=0$, que é o mesmo de que não há correlação entre o erro $v_{i}$ e as variáveis explicativas, tem importância fundamental, para que os estimadores sejam não viesados e consistentes (BACCHI E CALDARELLI, 2015).

De acordo com Hill, Griffiths e Judge (1999), já que existe correlação entre erros do mesmo indivíduo em períodos de tempos diferentes, os mínimos quadrados ordinários passam a não ser o método mais apropriado, sendo necessária então, a estimação de um método de mínimos quadrados generalizados (MQG), que apresenta melhores estimadores.

\subsection{TESTE DE HAUSMAN}

Para garantir a melhor especificação do modelo, ou seja, o uso do modelo adequado é necessário à utilização do teste de Hausman que foi descrito por Greene (2008), sendo utilizado então para definir entre os modelos fixos e aleatórios qual o mais apropriado.

O teste de Hausman define as seguintes hipóteses nula e alternativa:

$H_{0}=$ efeito aleatório, o mais apropriado; 
$H_{1}=$ efeito fixo, o mais apropriado

Em que $H_{0}: \operatorname{Cov}\left(a_{i}, X_{i t}=0\right.$ e $H_{1}: \operatorname{Cov}\left(a_{i t}, X_{i t} \neq 0\right.$.

A estatística de Hausman utilizada para testar as hipóteses é a seguinte:

$$
H=\left(b_{f e}-b_{\Re}\right)^{\prime}\left[\operatorname{Var}\left(b_{f e}\right)-\operatorname{Var}\left(b_{\mathfrak{R}}\right)\right]^{-1}\left(b_{f e}-b_{\mathfrak{R}}\right) \chi_{k}^{2}
$$

Onde:

$b_{f e}$ é o vetor dos estimadores do modelos com efeitos fixos;

$b_{\mathfrak{R}}$ é o vetor dos estimadores do modelos com efeitos aleatórios;

$\operatorname{Var}\left(b_{f e}\right)$ é a matriz de variâncias-covariâncias dos estimadores $b_{f e}$;

$\operatorname{Var}\left(b_{\Re}\right)$ é a matriz de variâncias-covariâncias dos estimadores $b_{\Re}$, ek é o número de regressores.

\subsection{DESCRIÇÃO DO BANCO DE DADOS}

A presente pesquisa foi realizada entre os anos de 2010 a 2015, envolvendo os municípios do Estado do Pará. Os dados foram coletados a partir de sítios oficiais do Governo Federal, em reais e deflacionados baseado pelo índice IPCA anual. Os dados para a variável dependente - PIBAGRO e PIBPERC - que serão explicadas pelas demais variáveis foram coletados no sítio do IBGE, nos anos de 2005 a 2015, recorte temporal do estudo e equivalem ao Valor Agregado Bruto da agropecuária a preços correntes de cada município paraense e o produto interno bruto per capita.

Para as variáveis PAMTEMP e PAMTERM referentes à produção agrícola municipal temporária e produção agrícola municipal permanente, os dados foram coletados no sítio do Sistema IBGE de Recuperação Automática (SIDRA), correspondente ao mesmo recorte temporal - 2010 a 2015 - que correspondem ao total da produção do produto das lavouras temporárias em reais.

A variável PRODPEC corresponde a produção de origem animal, por tipo de produto. Neste trabalho, o tipo de produto de origem animal utilizado foi o leite. Os 
dados coletados para essa variável também estão disponíveis no sítio do Sistema IBGE de Recuperação Automática e correspondem ao recorte temporal de 2010 a 2015.

Portanto, têm-se:

PIBAGRO, referente ao Valor Agregado Bruto;

PIBPERC, referente ao Produto Interno Bruto per capita;

PAMTEMP e PAMPERM, referentes às produções agrícolas temporária e permanentes, respectivamente;

PRODPEC, referente a produção pecuária de origem animal, o leite.

Para a primeira base de dados, foi estimado o modelo e realizado o teste de Hausman como indicado na literatura (GUJARATI, 2000), conforme demonstra a Tabela 01.

Tabela 01: Teste de Hausman.

\begin{tabular}{llll}
\hline Test Summary & Chi-Sq. Statistic & Chi-Sq. d.f. & Prob. \\
\hline Cross-section random & 34.073 .927 & 3 & 0.0000 \\
\hline
\end{tabular}

Fonte: Elaboração Própria com base nos dados do IBGE/SIDRA/FNDE.

De acordo com a Tabela 01 , o teste indica que a estimação deve assumir o modelo de efeitos fixos, uma vez que a probabilidade do teste foi de 0.0000 , o que ocasiona na rejeição da hipótese nula. Após a aplicação do teste, estimou-se a modelagem selecionada, gerando os resultados apresentados na Tabela 02.

Tabela 02: Estimação do modelo de dados em painel com efeitos aleatórios.

\begin{tabular}{|c|c|c|c|c|}
\hline Variable & Coefficient & Std. Error & t-Statistic & Prob. \\
\hline PAMPERM & 0.493510 & 0.076164 & 6.479 .604 & 0.0000 \\
\hline PAMTEMP & 0.253622 & 0.048484 & 5.231 .073 & 0.0000 \\
\hline PRODPEC & -0.147543 & 0.386061 & -0.382174 & 0.7024 \\
\hline C & 90634.33 & 2.494 .689 & 3.633 .091 & 0.0000 \\
\hline \multicolumn{5}{|c|}{$\begin{array}{l}\text { Effects Specification } \\
\text { Cross-section fixed (dummy variables) }\end{array}$} \\
\hline R-squared & 0.899959 & Mean dependent var & & 104554.2 \\
\hline Adjusted R-squared & 0.879474 & S.D. dependent var & & 113624.5 \\
\hline S.E. of regression & 39446.84 & Akaike info criterion & & 2.415 .771 \\
\hline Sum squared resid & $1.11 \mathrm{E}+12$ & Schwarz criterion & & 2.497 .081 \\
\hline Log likelihood & -10240.81 & Hannan-Quinn criter. & & 2.446 .899 \\
\hline F-statistic & 4.393.219 & Durbin-Watson stat & & 1.711 .032 \\
\hline Prob(F-statistic) & 0.000000 & & & \\
\hline
\end{tabular}


o que pode ser lido como oy $y_{i t}=90634.3_{0 i t}+0,49 P A M P E R M_{i t}+0,25 P A M T E M P_{i t}-$ $0,14 P R O D P E C_{i t}+e_{i t}$.

De acordo com a estimação, a variável PAMPERM que representa a produção agrícola municipal, com lavoura permanente apresenta significância estatística, ou seja, esta variável preditora, explicativa, afeta a variável dependente, alterando esta em $R$ \$ 0,49 , ou seja, o investimento de $R \$ 1,00$ nesta variável, vai gerar um incremento de $R \$$ 0,49 no PIBAGRO - Valor agregado bruto.

Já para a variável PAMTEMP que representa a produção agrícola municipal com lavoura temporária apresenta significância estatística, ou seja, esta variável independente, explicativa, afeta a variável dependente, alterando esta em $R \$ 0,25$, o que mostra que, o investimento de $R \$ 1,00$ nesta variável, vai gerar um incremento de $\mathrm{R} \$ 0,25$ no PIBAGRO - Valor agregado bruto.

De maneira contrária, as variáveis anteriores, a variável PRODPEC correspondente a produção pecuária, ou seja, o valor em real da produção de leite não apresenta significância estatística, então, o impacto que seria negativo passa a não existir, devido a probabilidade, que gera a não significância estatística desta.

Portanto, para a primeira estimação, onde procurou-se analisar os impactos no PIBAGRO, é possível observar que apenas as produções agrícola municipal, tanto as temporárias, quanto as lavouras permanentes causam impactos positivos na variável dependente PIBAGRO, diferente da produção pecuária, que não gera impactos.

Ainda de acordo com a estimação, o valor do $R^{2}$ corrobora com o apresentado, ou seja, a variável dependente é explicada pelas variações das variáveis independentes em aproximadamente $90 \%$, como também o teste $\mathrm{F}$ mostra-se significativo, para 0 modelo estimado.

Para a segunda base de dados, foi estimado o modelo e realizado o teste de Hausman para a escolha da modelagem, como recomendado na literatura (GUJARATI, 2000), conforme demonstra a Tabela 03, da mesma forma que foi realizado na primeira base de dados.

Tabela 03: Teste de Hausman

\begin{tabular}{llll}
\hline Test Summary & Chi-Sq. Statistic & Chi-Sq. d.f. & Prob. \\
Cross-section random & 12.991 .000 & 3 & 0.0045
\end{tabular}

Fonte: Elaboração Própria com base nos dados do IBGE/SIDRA/FNDE. 
De acordo com a Tabela 03, o teste indica que a estimação também deve assumir o modelo de efeitos fixos, uma vez que a probabilidade do teste foi de 0.0045 , o que ocasiona na não aceitação da hipótese nula e a aceitação da hipótese alternativa. Após a aplicação do teste, estimou-se a modelagem selecionada, gerando os resultados apresentados na Tabela 03.

A Tabela 04 demonstra a estimação do modelo de dados em painel com efeitos fixos, desta forma, o modelo $y_{i t}=\beta_{0 i t}+\beta_{1 i t} x_{1 i t}+\cdots+\beta_{\text {nit }} x_{k i t}+e_{i t}$, o passa a ser tido como $y_{i t}=12064,3_{0 i t}+0,02$ PAMPERM $i t+0,01$ PAMTEMP $i t+0,09 P R O D P E C_{i t}+e_{i t}$.

Tabela 04: Estimação do modelo de dados em painel com efeitos fixos.

\begin{tabular}{|c|c|c|c|c|}
\hline Variable & Coefficient & Std. Error & t-Statistic & Prob. \\
\hline PAMPERM & 0.022016 & 0.009451 & 2.329 .506 & 0.0201 \\
\hline PAMTEMP & 0.019323 & 0.006016 & 3.211 .737 & 0.0014 \\
\hline PRODPEC & 0.090401 & 0.047906 & 1.887 .055 & 0.0596 \\
\hline C & 12064.46 & 3.095 .613 & 3.897 .275 & 0.0000 \\
\hline \multicolumn{5}{|c|}{ Cross-section fixed (dummy variables) } \\
\hline R-squared & 0.924502 & Mean dependent var & & 13271.36 \\
\hline Adjusted R-squared & 0.909042 & S.D. dependent var & & 16230.13 \\
\hline S.E. of regression & 4.894 .885 & Akaike info criterion & & 1.998 .418 \\
\hline Sum squared resid & $1.71 \mathrm{E}+10$ & Schwarz criterion & & 2.079 .728 \\
\hline Log likelihood & -8.446 .197 & Hannan-Quinn criter. & & 2.029.547 \\
\hline F-statistic & 5.980 .084 & Durbin-Watson stat & & 1.305 .675 \\
\hline Prob(F-statistic) & 0.000000 & & & \\
\hline
\end{tabular}

Fonte: Elaboração Própria com base nos dados do IBGE/SIDRA/FNDE.

$\mathrm{Na}$ estimação este modelo, testou-se agora as variáveis independentes correspondentes as produções agrícolas permanentes, temporárias e a produção pecuária, porém, a variável dependente passa a ser o produto interno bruto per capita.

Diferente do modelo anterior em que apresentou a variável da produção pecuária negativa e sem significância estatística, neste modelo todas as variáveis foram positivas e com significância estatística, o que quer dizer que ambas são capazes de impactar positivamente no produto interno bruto per capita.

As variáveis PAMTEMP e PAMPERM por apresentarem significância estatística, causam assim impactos positivos na variável dependente $Y$. Portanto, tem-se que: 0 acréscimo de $R \$ 1,00$ de investimentos nas produções agrícolas municipais temporárias e permanentes, vai gerar um impacto positivo de $R \$ 0,02$ e $R \$ 0,01$ no Produto Interno Bruto per capita - PIB Per Capita - dos municípios, respectivamente. 
De forma análoga, o incremento de $R \$ 1,00$ na produção pecuária - produção de leite - causará um aumento de $\mathrm{R} \$ 0,09$ no Produto Interno Bruto per capita - PIB Per Capita - de cada município, ou seja, gera impactos positivos na economia do município.

No entanto, os impactos no Produto Interno Bruto per capita são inferiores aos impactos gerados no Valo Agregado Bruto - PIBAGRO. No entanto, nenhuma das três variáveis deixou de ter significância estatística, ou seja, todas elas são capazes de gerar impactos positivos na variável dependente.

O valor do $R^{2}$ corrobora o que foi exposto. O conjunto de variáveis preditoras possui um poder de explicação de mais de $90 \%$ da variável PIBPERC, sendo ainda demonstrado também nesse modelo que o resultado do teste $F$ é estatisticamente significativo.

\section{CONSIDERAÇÕES FINAIS}

A agricultura familiar é de grande importância no cenário econômico brasileiro. Além do fator econômico, a agricultura também tem papel fundamental na segurança alimentar da população. A falta de atividade econômica nesses setores pode causar impactos neutros ou até negativos na economia dos municípios, uma vez que o crescimento agrícola é capaz de constituir um importante vetor para a redução da pobreza.

O governo tem buscado fomentar o apoio à agricultura familiar incentivando a produção e a comercialização desses produtos provindos desse setor através de programas institucionais como o Programa Nacional de Alimentação Escolar (PNAE) e o Programa de Aquisição de Alimentos (PAA).

De acordo com o exposto, as estimações mostraram que em sua maioria, a produção agrícola temporária e permanente, como também a produção pecuária apresenta impactos positivos para a economia dos estados paraenses. Com relação ao Valor Agregado Bruto - PIB Agrícola - as produções agrícolas apresentam impactos positivos, já que apresentam significância estatística, diferente da produção pecuária, que por não ser significante estatisticamente, não gera impactos no PIB Agrícola. 
Quando a variável dependente passa a ser o Produto Interno Bruto per capita, todas as variáveis independentes passaram a causar impactos positivos, visto as suas significâncias estatísticas, ou seja, para a variável PIBPEC, os impactos econômicos nos municípios paraenses são todos positivos, porém, não são muito expressivos, chegando a valores como, por exemplo, $\mathrm{R} \$ 0,09$ centavos.

É possível que o baixo impacto econômico possa ter-se dado devido a alguns municípios não apresentarem atividade econômica em relação produção agrícola temporária, produção agrícola permanente e a produção pecuária, como também, é possível que esses valores sem muita expressão dê-se devido à participação mais elevada de outros setores como o setor industrial ou de serviços, na economia dos municípios, no entanto, esta pode ser outra etapa para um estudo futuro, no intuito de testar estas hipóteses levantadas.

Baseado no que foi exposto, fica clara a necessidade do investimento em políticas que fomentem a participação do agricultor familiar, facilitando a produção e a comercialização de seus produtos, contudo, é importante ressaltar que também é de suma importância o melhoramento tecnológico, para que estes produtores possam maximizar sua produção, gerando também produtos de qualidade que tenham plena capacidade de serem absorvidos pelos consumidores e pelo mercado.

\section{REFERÊNCIAS}

AVILA, M. L.; CALDAS, E. L.; ASSAD, S. S. Sinergia e coordenação em políticas públicas: o caso do PAA e PNAE. Sociedade E Desenvolvimento Rural on line. v.7, n. 3, jul. 2013.

BACCHI, M. R. P.; CALDARELLI, C. E.; Impactos socioeconômicos da expansão do setor sucroenergético no Estado de São Paulo, entre 2005 e 2009. Nova Economia (UFMG), v. 25, p. 209-224, 2015.

\section{BRASIL. PROGRAMA DE AQUISIÇÃO DE ALIMENTOS DA AGRICULTURA} FAMILIAR: Renda para quem produz e comida na mesa de quem precisa. Ministério do Desenvolvimento Agrário. 2012. <http://www.mda.gov.br/sitemda/sites/sitemda/files/user_arquivos_64/CARTILHA_PAA_ FINAL.pdf>. Acesso em: 02 abr. 2018. 
BRASIL. PNAE. Disponível em:

<http://www.fnde.gov.br/dadosabertos/organization/about/pnae>. Acesso em: 01 nov. 2017.

BRASIL. Ministério da Educação. Resolução n. 38, de 16 de julho de 2009. Fundo Nacional de Desenvolvimento da Educação, Brasília, DF, 16 de julho de 2009. Disponível em: <ftp://ftp.fnde.gov.br/web/resolucoes_2009/res038_16072009.pdf>. Acesso em: 01 nov. 2017.

CARVALHO, F. J. C de.; Keynes e o Brasil. Economia e Sociedade (UNICAMP), v. 17, p. $569-574,2008$.

DUARTE, P. C.; LAMOUNIER, W.M.; TAKAMATSU R.T.; Modelos econométricos para dados em painel: aspectos teóricos e exemplos de aplicação à pesquisa em contabilidade e finanças. In: CONGRESSO USP DE INICIAÇÃO CIENTÍFICA EM CONTABILIDADE, 4., 2007, São Paulo. Anais... FEA-USP, São Paulo, 2007.

FAPESPA. BOLETIM AGROPECUÁRIO DO ESTADO DO PARÁ 2015. Fundação Amazônia de Amparo a Estudos e Pesquisas do Pará Boletim Agropecuário do Estado do Pará 2015. Belém, n. 1, jul. 2015. 38 f.

FNDE. Dados da agricultura familiar. Disponível em: $<$ http://www.fnde.gov.br/programas/pnae/pnae-consultas/pnae-dados-da-agriculturafamiliar>. Acesso em 13 fev. 2018.

GREENE, W.H. Econometric Analysis. 6. ed. New Jersey: Prentice Hall, 2008. 1178 p.

GUJARATI, D. N. Econometria Básica. 3 ed. São Paulo: Makron Books, 2000. 846 p.

HELLER, C. A síntese da Teoria Geral do Emprego, dos Juros e da Moeda segundo Roy Harrod em "Mr. Keynes and traditional theory". Revista de Economia, Curitiba: Universidade Federal do Paraná, v. 25, n. 23, p.27-49, 2000.

HILL, R. C.; GRIFFITHS, W. E.; JUDGE, G. G. Econometria. São Paulo: Saraiva, 1999.

HSIAO, C.; Analysis of panel data. Cambridge: Cambridge University Press, 1986.

; Panel Data Analysis - Advantages and Challenges, invited key paper, TEST, 16, 2007, 1-22.

HOFFMAN, R. A agricultura familiar produz $70 \%$ dos alimentos consumidos no Brasil? Segurança Alimentar e Nutricional, Campinas, v. 21, n. 1, p. 417-421, 2014.

IPEA. Agricultura: evolução e importância para a balança comercial brasileira. Texto para discussão / Instituto de Pesquisa Econômica Aplicada. Brasília: Rio de Janeiro: Ipea, 2014. 
LÁU, H. D. Pecuária no Estado do Pará: índices, limitações e potencialidades. Hugo Didonet Láu. Belém, PA: Embrapa Amazônia Oriental, 2006. 36p.

MEDEIROS NETO, J. B. Desafio à pecuária brasileira. Porto Alegre: Sulina, 1970. $215 \mathrm{p}$.

PARAÍBA. Sedh acompanha e monitora Programa do Leite da Paraíba. 2017. <http://paraiba.pb.gov.br/sedh-acompanha-e-monitora-programa-do-leite-da-paraiba/>. Acesso em: 01 abr. 2018.

SILVA, C. V. A da.; Desafios para a ampliação da participação da agricultura familiar no programa nacional de alimentação escolar na região do baixo tocantins, Pará. $101 \mathrm{f}$. Dissertação (Mestrado em Desenvolvimento Rural e Gestão de Empreendimentos Agroalimentares) - Instituto Federal de Educação, Ciência e Tecnologia do Pará IFPA, 2017.

SOUZA, S. F de; SOUZA, A. L de; SANTOS, R. B. N; BORJA, O. R. P.; FERREIRA, F. D. S.; PAULINO, G. S.; Eficiência nos gastos públicos com a merenda escolar no Pará: um comparativo entre 2011 e 2014. In: COLÓQUIO MERCADO INSTITUCIONAL DE ALIMENTOS E ECONOMIA SOLIDÁRIA, 4., 2017, Maringá - PR. Anais eletrônics... Maringá, 2017.

STOCK, J., WATSON, M. Introduction to Econometrics. 2.ed. São Paulo: Pearson, 2007. 